\title{
Sommes sans grand facteur premier
}

\author{
par \\ R. DE LA Bretèche (Orsay)
}

1. Introduction. De nombreuses questions d'arithmétique se posent en termes d'indépendance entre les structures additive et multiplicative de l'ensemble des entiers. Une mesure efficace de cette indépendance est fournie par l'étude de la valeur moyenne de l'exponentielle - c'est-à-dire de $e(\theta n):=\exp (2 \pi i \theta n)$ - sur une suite d'entiers définie multiplicativement. Ainsi le célèbre théorème de Vinogradov [16] affirme que

$$
\lim _{x \rightarrow \infty} \pi(x)^{-1} \sum_{p \leq x} e(\vartheta p)=0
$$

pour tout $\vartheta$ irrationnel, où $p$ désigne un nombre premier générique. De même, Dupain, Hall et Tenenbaum obtiennent ce type de résultat pour la suite des entiers ayant un nombre fixé de facteurs premiers ([5], [14]). Soit $\Omega(n)$ le nombre de facteurs premiers comptés avec multiplicité d'un entier générique $n$. Ils établissent ainsi, pour toute constante $\delta \in] 0,2[$, la relation

$$
\lim _{x \rightarrow \infty}\left(\sup _{k \leq(2-\delta) \log _{2} x}\left(\frac{\sum_{n \leq x, \Omega(n)=k} e(\vartheta n)}{|\{n \leq x: \Omega(n)=k\}|}\right)\right)=0
$$

pour tout $\vartheta$ irrationnel où l'on désigne par $|\mathcal{A}|$ le cardinal de l'ensemble $\mathcal{A}$ et par $\log _{2}$ la deuxième itérée de la fonction logarithme.

Nous nous proposons d'étudier le cas de la suite des entiers sans grand facteur premier. Désignons par $P(n)$ le plus grand facteur premier d'un entier générique $n$, avec la convention $P(1)=1$. L'ensemble

$$
S(x, y):=\{n \leq x: P(n) \leq y\}
$$

a fait ces dix dernières années l'objet de nombreux travaux. Le lecteur trouvera une abondante bibliographie dans l'article de Hildebrand et Tenenbaum [10]. Soit $u:=\log x / \log y$. Hildebrand [9] a établi la validité de

1991 Mathematics Subject Classification: 11N25, 11L69. 
l'approximation

$$
\Psi(x, y):=|S(x, y)|=x \varrho(u)\left\{1+O\left(\frac{\log (u+1)}{\log y}\right)\right\}
$$

dans le domaine

$$
x \geq 3, \quad \exp \left\{\left(\log _{2} x\right)^{5 / 3+\varepsilon}\right\} \leq y \leq x
$$

pour $\varepsilon>0$ fixé, où $\varrho$ désigne la fonction de Dickman, l'unique solution continue à droite sur $\mathbb{R}$ de l'équation différentielle aux différences

$$
\begin{aligned}
u \varrho^{\prime}(u) & =-\varrho(u-1) & & (u \geq 1), \\
\varrho(u) & =1 & & (0 \leq u \leq 1), \\
\varrho(u) & =0 & & (u<0) .
\end{aligned}
$$

Le lecteur trouvera une étude complète de la fonction de Dickman dans le livre de Tenenbaum [15] (paragraphe III.5.5.4).

Posons

$$
E(x, y ; \vartheta):=\sum_{n \in S(x, y)} e(\vartheta n) .
$$

G. Tenenbaum a établi un résultat effectif de (2) uniformément valable pour tout $\vartheta \in \mathbb{R}:$ on a uniformément pour $x \geq 1, k \geq 1, \vartheta \in \mathbb{R}$,

$$
\sum_{\substack{n \leq x \\ \Omega(n)=k}} e(\vartheta n)=\frac{|\{n \leq x: \Omega(n)=k\}|}{x}\left\{E(x, x ; \vartheta)+O\left(x \delta_{k}(x)\right)\right\},
$$

avec

$$
\delta_{k}(x):=\frac{1}{\sqrt{\log x}}+\frac{\left|k-\log _{2} x\right|}{\log _{2} x} .
$$

Cette estimation est non triviale que pour $k \sim \log _{2} x$, et la précision de la formule ne peut être améliorée en gardant l'uniformité en $\vartheta$.

Nous utilisons la même démarche pour montrer le résultat suivant.

ThÉORÈme 1. On a uniformément pour $\vartheta \in \mathbb{R}$ et $(x, y)$ satisfaisant à

$$
x \geq 3, \quad \exp \left\{(\log x)^{2 / 3+\varepsilon}\right\} \leq y
$$

l'évaluation

$$
E(x, y ; \vartheta)=\varrho(u)\left\{E(x, x ; \vartheta)+O\left(x \frac{\log (u+1)}{\log y}\right)\right\} .
$$

Soit

$$
\Delta(x, y ; \vartheta):=E(x, y ; \vartheta)-\varrho(u) E(x, x ; \vartheta)
$$


Le majorant de $\sup _{\vartheta \in \mathbb{R}}|\Delta(x, y ; \vartheta)|$ ne peut pas être choisi plus petit que la quantité $\Psi(x, y) \log (u+1) / \log y$ puisque l'on a (voir [4])

$$
\Delta(x, y ; 1 / 2) \gg \Psi(x, y) \frac{\log (u+1)}{\log y} \quad\left((x, y) \text { vérifiant }\left(G_{\varepsilon}\right)\right) .
$$

Ici, la difficulté pour établir une telle formule est due à l'uniformité par rapport à $\vartheta$. Nous verrons ici que cette uniformité permet de démontrer de nouveaux résultats.

Pour des résultats complémentaires sur $E(x, y ; \vartheta)$, nous renvoyons le lecteur à l'article de l'auteur [4] qui améliore et complète le travail de Fouvry et Tenenbaum [8]. Nous citons un de ses résultats. Soient $\vartheta \in \mathbb{R} \backslash \mathbb{Z}$ et $Q \geq 2$. Le théorème de Dirichlet affirme qu'il existe un rationnel $a / q$ tel que $(a, q)=1,1 \leq q \leq Q$ et

$$
\left|\vartheta-\frac{a}{q}\right| \leq \frac{1}{q Q} .
$$

Le dénominateur $q$ n'est pas défini de manière unique. Soit $q(\vartheta ; Q)$ le plus petit dénominateur vérifiant (7). Le corollaire 4 de [4] permet d'affirmer que lorsque $q:=q\left(\vartheta ; x \exp \left\{-c_{2} \sqrt{\log y}\right\}\right) \geq 2, u \geq \omega(q)+1$, il existe une constante $M>0$ telle que, pour tout $\kappa>0$ fixé, la majoration

$$
E(x, y ; \vartheta) \ll \Psi(x, y) \frac{\log q}{\phi(q)}\left(\frac{M \log (u+1)}{\log y}\right)^{\omega(q)}+\frac{\Psi(x, y)}{(\log y)^{\kappa}}
$$

soit uniformément valable par rapport à $(x, y)$ satisfaisant à $\left(G_{\varepsilon}\right)$.

Cette indépendance entre la structure multiplicative et additive peut aussi être étudiée en regardant les propriétés multiplicatives de la somme de deux ensembles. Soit $\mathcal{A}$ et $\mathcal{B}$ deux sous-ensembles non vides de $[1, x] \cap \mathbb{N}$. Nous désignons par somme directe de $\mathcal{A}$ et $\mathcal{B}$, et nous notons $\mathcal{A} \oplus \mathcal{B}$, la suite finie d'entiers dont les éléments sont ceux de $\mathcal{A}+\mathcal{B}$ mais où chaque terme apparaît autant de fois qu'il est représentable sous la forme $a+b, a \in \mathcal{A}$, $b \in \mathcal{B}$. On a donc $|\mathcal{A} \oplus \mathcal{B}|=|\mathcal{A}| \cdot|\mathcal{B}|$. Ce sujet a donné lieu ces dix dernières années à de nombreux travaux ([1], [2], [7], [6], [13], [14]). G. Tenenbaum montre dans [14], à l'aide de (2), le résultat suivant.

ThÉorème A (Tenenbaum). Soient $\mathcal{A}$ et $\mathcal{B}$ deux sous-ensembles non vides de $[1, x] \cap \mathbb{N}$. Pour $x \geq 3, k \geq 1$, on a

$$
\frac{1}{|\mathcal{A}| \cdot|\mathcal{B}|} \sum_{\substack{n \in \mathcal{A} \oplus \mathcal{B} \\ \Omega(n)=k}} 1=\frac{|\{n \leq x: \Omega(n)=k\}|}{x}\left\{1+O\left(\frac{x \delta_{k}(x)}{\sqrt{|\mathcal{A}| \cdot|\mathcal{B}|}}\right)\right\} .
$$

Nous allons étudier la répartition des entiers sans grand facteur premier dans la somme directe $\mathcal{A} \oplus \mathcal{B}$. Le résultat suivant qui découle facilement du Théorème 1 affirme que la densité des entiers sans grand facteur premier de 
la suite $\mathcal{A} \oplus \mathcal{B}$ est aussi donnée par la fonction de Dickman dans un large domaine.

ThÉORÈme 2 . Soient $\mathcal{A}$ et $\mathcal{B}$ deux sous-ensembles non vides de $[1, x] \cap \mathbb{N}$. Pour $(x, y)$ satisfaisant $\grave{a}\left(G_{\varepsilon}\right)$, on a

$$
\frac{1}{|\mathcal{A}| \cdot|\mathcal{B}|} \sum_{\substack{n \in \mathcal{A} \oplus \mathcal{B} \\ P(n) \leq y}} 1=\varrho(u)\left\{1+O\left(\frac{x}{\sqrt{|\mathcal{A}| \cdot|\mathcal{B}|}} \cdot \frac{\log (u+1)}{\log y}\right)\right\} .
$$

Ce résultat est de nature différente de ceux énoncés en [1]-[3]. Seul l'existence d'entier dans $\mathcal{A} \oplus \mathcal{B}$ ayant un plus grand facteur premier de l'ordre de $|\mathcal{A} \oplus \mathcal{B}|$ était établie. Ici, on détermine précisément le nombre de ces entiers $n$ tels que $P(n) \leq y$ en fournissant un terme principal du membre de gauche de (10) lorsque $y$ décrit un large domaine.

Le terme d'erreur de (10) dans sa généralité est optimal puisque le cas $\mathcal{A}=\mathcal{B}=\mathbb{N} \cap[1, x]$ ne tolère pas un terme d'erreur plus petit. Cette précision permet de retrouver le résultat de Balog et Sárközy [2], amélioré par la suite par Sárközy et Stewart [12], qui établissent notamment que, pour $x$ suffisamment grand, $\max _{n \in \mathcal{A} \oplus \mathcal{B}} P(n) \gg x$ lorsque que $\mathcal{A}$ et $\mathcal{B}$ sont des ensembles denses, i.e. $|\mathcal{A}| \gg x$ et $|\mathcal{B}| \gg x$. En effet, considérons le nombre d'entiers $n$ de $\mathcal{A} \oplus \mathcal{B}$ tels que $P(n) \leq c x$. Grâce à (10), nous pouvons montrer que, pour une constante suffisamment petite $c<1$, il y en a strictement moins que $|\mathcal{A}| \cdot|\mathcal{B}|$.

Notre résultat est particulièrement performant lorsque les ensembles $\mathcal{A}$ et $\mathcal{B}$ sont denses. Le cardinal de l'ensemble des entiers de $\mathcal{A} \oplus \mathcal{B}$ dont tous les facteurs premiers sont inférieur à $y$ est équivalent à $\varrho(u)$ dès que

$$
\frac{|\mathcal{A}| \cdot|\mathcal{B}|}{x^{2}} \cdot \frac{(\log y)^{2}}{(\log (u+1))^{2}} \rightarrow \infty \quad\left(x \rightarrow \infty, \quad(x, y) \text { vérifiant }\left(G_{\varepsilon}\right)\right) .
$$

Il serait intéressant de déterminer avec précision dans quelle mesure cela reste vrai pour des ensembles $\mathcal{A}$ et $\mathcal{B}$ moins denses, respectivement pour des couples $(x, y)$ ne satisfaisant pas aux inégalités $\left(G_{\varepsilon}\right)$.

Pour des ensembles moins denses, Sárközy et Stewart ont montré qu'il existe des constantes $c_{1}, c_{2}$ telles que pour $|\mathcal{A}| \cdot|\mathcal{B}| \gg x^{5 / 3+\varepsilon}$ il existe au moins $c_{1}|\mathcal{A}| \cdot|\mathcal{B}| / \log x$ couples $(a, b)$ satisfaisant à l'inégalité

$$
c_{2} \frac{|\mathcal{A}|^{1 / 2} \cdot|\mathcal{B}|^{1 / 2}}{(\log R) \log _{2} R} \leq P(a+b) \leq 2 c_{2} \frac{|\mathcal{A}|^{1 / 2} \cdot|\mathcal{B}|^{1 / 2}}{(\log R) \log _{2} R}, \quad R:=3 \frac{x}{\sqrt{|\mathcal{A}| \cdot|\mathcal{B}|}}
$$

D'autre part, nous exhibons ici un contre-exemple qui montre qu'une formule générale de type (10) n'est plus valable lorsque l'on a

$$
\frac{|\mathcal{A}| \cdot|\mathcal{B}|}{x^{2}} \ll \frac{1}{y^{2}} .
$$


En effet en prenant $\mathcal{A}=\mathcal{B}=\{n \leq x: p(y) \mid n\}$ où on a noté $p(y)$ le plus petit nombre premier compris entre $y+1$ et $2 y+2$, on a la relation (12) et

$$
\sum_{\substack{n \in \mathcal{A} \oplus \mathcal{B} \\ P(n) \leq y}} 1=0
$$

puisque pour tout $a \in \mathcal{A}$ et pour tout $b \in \mathcal{B}$ on a $p(y) \mid a+b$.

Sárközy et Stewart remarquent dans [12] qu'une conséquence de l'estimation (11) est qu'il existe une constante $C>0$ pour des ensembles $\mathcal{A}$ et $\mathcal{B}$ denses telle que pour tout $y \in\left[x^{5 / 6+\varepsilon}, C x\right]$, le nombre d'entiers $n$ de $\mathcal{A} \oplus \mathcal{B}$ vérifiant $y \leq P(n) \leq 3 y$ est de l'ordre de grandeur attendu. Nous obtenons directement du Théorème 2 le résultat suivant.

Corollaire 2.1. Soient les ensembles $\mathcal{A} \subset[1, x] \cap \mathbb{N}$ et $\mathcal{B} \subset[1, x] \cap \mathbb{N}$ et une constante $\delta>0$ tels que $|\mathcal{A}| \cdot|\mathcal{B}| \geq \delta x^{2}$. On fixe $\varepsilon>0$. Il existe des constantes $C^{\prime}=C^{\prime}(\delta)>1$ et $0<C^{\prime \prime}=C^{\prime \prime}(\delta)<1$ telles que pour tout couple $(x, y)$ satisfaisant $\grave{a}$

$$
x \geq x_{0}, \quad \exp (\log x)^{2 / 3+\varepsilon} \leq y \leq C^{\prime \prime} x,
$$

on ait

$$
\frac{1}{|\mathcal{A}| \cdot|\mathcal{B}|}\left|\left\{n \in \mathcal{A} \oplus \mathcal{B}: P(n) \in\left[y, C^{\prime} y\right]\right\}\right| \gg \frac{u\left|\varrho^{\prime}(u)\right|}{\log y} .
$$

Remarques. (i) Lorsque $u \rightarrow \infty$, le membre de gauche de (13) est, en fait, équivalent à

$$
-\left(\log C^{\prime}\right) u \varrho^{\prime}(u) / \log y
$$

(ii) Le membre de droite de (13) est du même ordre de grandeur que la quantité $Q\left(x, C^{\prime} y, y\right) / x$ où l'on a posé

$$
Q(x, z, y):=\Psi(x, z)-\Psi(x, y)=\mid\{n \leq x: P(n) \in] y, z]\} \mid \quad(z \geq y) .
$$

En effet, pour $x \geq y^{2}$ et $(x, y)$ satisfaisant à $\left(G_{\varepsilon}\right)$, d'après [11], on a

$$
\Psi(x, y)=x \varrho(u)+x \frac{\varrho^{\prime}(u)}{\log y}+O\left(x \frac{\varrho(u)(\log (u+1))^{2}}{(\log y)^{2}}\right) \quad\left((x, y) \in\left(G_{\varepsilon}\right)\right) .
$$

En utilisant les relations

$$
\begin{aligned}
\varrho^{\prime \prime}(u-v) & \ll \varrho(u)(\log (u+1))^{2} \\
\varrho^{\prime}(u-v)-\varrho^{\prime}(u) & \ll v \varrho(u)(\log (u+1))^{2} \quad(v \log (u+1) \ll 1),
\end{aligned}
$$

on en déduit la relation valable pour $x \geq y^{2}$ :

$$
Q\left(x, C^{\prime} y, y\right)=-x\left(\log C^{\prime}\right) \frac{u \varrho^{\prime}(u)}{\log y}+O\left(x \frac{u^{2}(\log (u+1))^{2} \varrho(u)}{(\log y)^{2}}\right) .
$$


On désigne la partie entière d'un nombre réel $t$ par $[t]$. Pour $C^{\prime \prime-1} y \leq x<y^{2}$, on a

$$
Q\left(x, C^{\prime} y, y\right)=\sum_{y<p \leq C^{\prime} y} \sum_{n \leq x / p} 1=\sum_{y<p \leq C^{\prime} y}\left[\frac{x}{p}\right] .
$$

Le théorème des nombres premiers implique alors

$$
\begin{aligned}
Q\left(x, C^{\prime} y, y\right) & =x \frac{\log C^{\prime}}{\log y}+O\left(\frac{y}{\log y}+\frac{x}{(\log y)^{2}}\right) \\
& =x \frac{u \varrho^{\prime}(u)}{\log y}\left(-\left(\log C^{\prime}\right)+O\left(C^{\prime} C^{\prime \prime}+1 /(\log y)\right)\right),
\end{aligned}
$$

où l'on a utilisé la relation $u \varrho^{\prime}(u)=-1$ pour tout $1 \leq u \leq 2$. En choisissant la constante $C^{\prime \prime}$ suffisamment petite par rapport à $1 / C^{\prime}$, on obtient

$$
Q\left(x, C^{\prime} y, y\right) \asymp x \frac{-u \varrho^{\prime}(u)}{\log y} .
$$

C'est un devoir et un plaisir d'exprimer ma gratitude à A. Sárközy et G. Tenenbaum pour leurs conseils, leurs suggestions et leurs encouragements. Qu'ils soient ici remerciés!

\section{Démonstration des résultats}

2.1. Démonstration du Théorème 1. Nous utiliserons tout au long de la démonstration la notation $Y:=\exp \sqrt{\log y}$ et $\|\vartheta\|:=\min \{\vartheta-[\vartheta]$, $[\vartheta+1]-\vartheta\}$ la distance d'un nombre réel $\vartheta$ à l'ensemble des entiers. Nous étudions $E(x, y ; \vartheta)$ en fonction des bonnes approximations rationnelles de $\vartheta$. Soit $Q:=\left[x Y^{-c}\right]$ où $c$ sera une constante choisie suffisamment petite. Le théorème de Dirichlet affirme qu'il existe un couple d'entiers $(a, q)$ premiers entre eux tel que

$$
1 \leq q \leq Q, \quad|\vartheta-a / q| \leq 1 /(q Q) .
$$

Lorsque $q \geq 2$, on a d'après le Théorème 2 de [4] la majoration

$$
|E(x, y ; \vartheta)| \ll \Psi(x, y) \frac{\log (u+1)}{\log y}, \quad(x, y) \text { satisfaisant à }\left(G_{\varepsilon}\right) .
$$

Le domaine de validité de cette majoration est actuellement le plus large connu. Un élargissement de celui-ci impliquerait un élargissement de celui de la validité des estimations (10) et (13).

Or lorsque $q \geq 2$, on a la formule

$$
\varrho(u) E(x, x ; \vartheta) \ll \frac{\Psi(x, y)}{\|\vartheta\| x} \ll \frac{\Psi(x, y)}{Y^{c}} .
$$

On en déduit la majoration de $\Delta(x, y ; \vartheta)$ recherchée pour $q \geq 2$. 
Il reste à examiner le cas $q=1$. On a alors

$$
\|\vartheta\| \leq 1 / Q \text {. }
$$

En vue d'applications ultérieures, nous démontrons le résultat suivant valable lorsque $\|\vartheta\| \leq 1 / Q$ qui implique l'estimation du Théorème 1 .

Proposition 1. Soit $c>0$ une constante choisie suffisamment petite. Pour tout $(x, y)$ satisfaisant à $\left(G_{\varepsilon}\right)$ et $\vartheta \in \mathbb{R}$ tel que $\|\vartheta\| \leq Y^{c} / x$, on a

$$
E(x, y ; \vartheta)=\varrho(u) E(x, x ; \vartheta)+O(R(x, y ; \vartheta))
$$

avec

$$
R(x, y ; \vartheta):=\Psi(x, y) \frac{\log (u+1)}{\log y} \cdot \frac{\log (1+x\|\vartheta\|)}{x\|\vartheta\|}+\frac{\Psi(x, y)}{Y^{c}} .
$$

REmarque. Il est à noter que

$$
\frac{\log (1+x\|\vartheta\|)}{x\|\vartheta\|} \asymp \min \left\{1, \frac{\log (2+x\|\vartheta\|)}{x\|\vartheta\|}\right\} .
$$

Démonstration (de la Proposition 1). Cette relation est évidente pour $x \leq y$, nous nous restreignons donc par la suite au cas $x \geq y$. Ici, nous utilisons toute la précision de l'approximation $\Lambda(x, y)$ de de Bruijn de la quantité $\Psi(x, y)$ établie dans $\left(H_{\varepsilon}\right)$ par Saias:

$$
\Psi(x, y)=\Lambda(x, y)+O\left(\Psi(x, y) Y^{-2 c}\right)
$$

où

$$
\Lambda(x, y):= \begin{cases}x \int_{0-}^{\infty} \varrho(u-v) d\left(\left[y^{v}\right] y^{-v}\right) & (x \notin \mathbb{Z}), \\ \Lambda(x+, y) & (x \in \mathbb{Z}) .\end{cases}
$$

Par sommation d'Abel, on a aussi

$$
\Lambda(x, y)=x \varrho(u)-\{x\}-x \int_{0}^{u-1} \varrho^{\prime}(u-v)\left\{y^{v}\right\} y^{-v} d v .
$$

Montrons qu'il est suffisant d'estimer $E(x, y ; \vartheta)-E\left(x Y^{-c}, y ; \vartheta\right)$. L'évaluation (3) et la relation $\varrho(u-v) \ll \varrho(u)$ valable tant que $v \log u \ll 1$ (voir le livre de Tenenbaum [15], Corollaire III.5.8.4) implique dans $\left(G_{\varepsilon}\right)$ la validité de la majoration

$$
\varrho\left(u\left(x Y^{-c}\right)\right)=\varrho(u-c / \sqrt{\log y}) \ll \varrho(u) .
$$

On en déduit

$$
\Psi\left(\frac{x}{Y^{c}}, y\right) \ll \frac{x}{Y^{c}} \varrho(u) \ll \frac{\Psi(x, y)}{Y^{c}} .
$$

Ainsi $E\left(x Y^{-c}, y ; \vartheta\right)$ peut être englobé dans le terme d'erreur de (16). 
Quitte à évaluer la quantité conjuguée, nous imposons $\vartheta=\|\vartheta\|$. On a

$$
\begin{aligned}
E(x, y ; \vartheta)-E\left(\frac{x}{Y^{c}}, y ; \vartheta\right) & =\int_{x Y^{-c}}^{x} e(\vartheta t) d \Psi(t, y) \\
& =\int_{x Y^{-c}}^{x} e(\vartheta t) d\left\{\Lambda(t, y)+O\left(\Psi(t, y) Y^{-2 c}\right)\right\}
\end{aligned}
$$

Une intégration par parties et l'inégalité (15) montrent que la contribution du terme d'erreur est majoré par $\Psi(x, y) Y^{-2 c}(1+\|\vartheta\| x) \ll \Psi(x, y) Y^{-c}$, ce qui est acceptable.

Notons $u(t):=\log t / \log y$. Un simple calcul à partir de (18) fournit l'égalité entre mesures

$$
\begin{aligned}
d \Lambda(t, y)= & \varrho(u(t)) d t+\frac{\varrho^{\prime}(u(t))}{\log y} d t+\frac{y}{\log y} \cdot \frac{\{t / y\}}{t} d t-d\{t\} \\
& -\int_{0}^{u-1}\left(\varrho^{\prime}(u-v)+\frac{\varrho^{\prime \prime}(u-v)}{\log y}\right) \frac{\left\{y^{v}\right\}}{y^{v}} d v d t .
\end{aligned}
$$

Il s'agit maintenant d'évaluer la contribution de chaque terme de cette expression à l'intégrale $\int_{x Y^{-c}}^{x} e(\vartheta t) d \Lambda(t, y)$.

La fonction $t \mapsto \varrho(u(t))$ est dérivable par morceaux sur $\left[x Y^{-c}, x\right]$ puisque l'on a supposé $x \geq y$. On imposera $\varrho^{\prime}(1)=-1$ afin que $\varrho^{\prime}$ soit continue à droite et que la relation $u \varrho^{\prime}(u)=-\varrho(u-1)$ soit vérifiée en $u=1$. Grâce à une intégration par parties, on obtient donc

$$
\begin{aligned}
\int_{x Y^{-c}}^{x} e(\vartheta t) \varrho(u(t)) d t & \\
& =\left[\frac{e(\vartheta t)-1}{2 \pi i\|\vartheta\|} \varrho(u(t))\right]_{x Y^{-c}}^{x}-\int_{x Y^{-c}}^{x} \frac{e(\vartheta t)-1}{2 \pi i\|\vartheta\|} \cdot \frac{\varrho^{\prime}(u(t))}{t \log y} d t .
\end{aligned}
$$

Utilisons la formule asymptotique

$$
E(t, t ; \vartheta)=\frac{e(\vartheta t)-1}{2 \pi i\|\vartheta\|}+O(1) \ll \min \left\{t, \frac{1}{\|\vartheta\|}\right\} .
$$

Il vient

$$
\begin{aligned}
{\left[\frac{e(\vartheta t)-1}{2 \pi i\|\vartheta\|} \varrho(u(t))\right]_{x Y^{-c}}^{x} } & =\varrho(u)(E(x, x ; \vartheta)+O(1))+O\left(\frac{x}{Y^{c}} \varrho\left(u\left(x Y^{-c}\right)\right)\right) \\
& =\varrho(u) E(x, x ; \vartheta)+O\left(\frac{\Psi(x, y)}{Y^{c}}\right),
\end{aligned}
$$

où l'on a utilisé (19). 
En utilisant la majoration $\varrho^{\prime}(v) \ll \varrho(v) \log (v+1)(v \geq 0)$ (voir le Corollaire III.5.8.3 de [15]), la décroissance de $\varrho$ et (19), puis (22), nous obtenons

$$
\int_{x Y^{-c}}^{x} \frac{e(\vartheta t)-1}{2 \pi i\|\vartheta\|} \cdot \frac{\varrho^{\prime}(u(t))}{t \log y} d t \ll \frac{\varrho(u) \log (u+1)}{\log y} \int_{x Y^{-c}}^{x} \min \left\{1, \frac{1}{\|\vartheta\| t}\right\} d t .
$$

Après avoir remarqué que

$$
\int_{\max \left\{x Y^{-c}, y\right\}}^{x} \min \left\{1, \frac{1}{\|\vartheta\| t}\right\} d t \ll x \min \left\{1, \frac{\log (\|\vartheta\| x+2)}{\|\vartheta\| x}\right\},
$$

nous obtenons

$$
\int_{x Y^{-c}}^{x} \frac{e(\vartheta t)-1}{2 \pi i\|\vartheta\|} \cdot \frac{\varrho^{\prime}(u(t))}{t \log y} d t \ll x \varrho(u) \frac{\log (u+1)}{\log y} \min \left\{1, \frac{\log (\|\vartheta\| x+2)}{\|\vartheta\| x}\right\} .
$$

Nous en déduisons donc que

$$
\int_{x Y^{-c}}^{x} e(\vartheta t) \varrho(u(t)) d t=\varrho(u) E(x, x ; \vartheta)+O(R(x, y ; \vartheta)) .
$$

Il reste à montrer que la contribution de tous les autres termes peut être englobée par le terme d'erreur de (16).

Posons $f$ la fonction définie par $f(t)=1$ lorsque $t \in]-\infty, 1]$ et $f(t)=0$ lorsque $t \in] 1, \infty\left[\right.$ de sorte que la fonction $t \mapsto \varrho^{\prime}(t)+f(t)$ soit continue sur $\mathbb{R}$ et dérivable par morceaux. Une intégration par parties fournit

$$
\begin{aligned}
& \text { 25) } \int_{x Y^{-c}}^{x} e(\vartheta t) \frac{\varrho^{\prime}(u(t))+f(u(t))}{\log y} d t \\
& =\left[\frac{e(\vartheta t)-1}{2 \pi i\|\vartheta\|} \cdot \frac{\varrho^{\prime}(u(t))+f(u(t))}{\log y}\right]_{x Y^{-c}}^{x}-\int_{x Y^{-c}}^{x} \frac{e(\vartheta t)-1}{2 \pi i\|\vartheta\|} \varrho^{\prime \prime}(u(t)) \frac{d t}{t(\log y)^{2}} .
\end{aligned}
$$

Les mêmes manipulations que dans la démonstration de (24) permettent de montrer que le membre de gauche de $(25)$ est $O(R(x, y ; \vartheta))$. De plus, on a

$$
\begin{aligned}
\int_{x Y^{-c}}^{x} e(\vartheta t) \frac{f(u(t))}{\log y} d t & =\frac{1}{\log y} \int_{\max \left\{x Y^{-c}, y\right\}}^{y} e(\vartheta t) d t \\
& =\frac{1}{\log y}\left[\frac{e(\vartheta t)-1}{2 \pi i\|\vartheta\|}\right]_{\max \left\{x Y^{-c}, y\right\}}^{y} \\
& \leq \frac{1}{\log y} \min \left\{\|\vartheta\|^{-1}, y\right\} .
\end{aligned}
$$

Puisque ce terme est nul pour $x Y^{-c} \geq y$, on a 


$$
\int_{x Y^{-c}}^{x} e(\vartheta t) \frac{f(u(t))}{\log y} d t \ll R(x, y ; \vartheta) .
$$

On en déduit la majoration

$$
\int_{x Y^{-c}}^{x} e(\vartheta t) \frac{\varrho^{\prime}(u(t))}{\log y} d t \ll R(x, y ; \vartheta) .
$$

Une intégration par parties fournit

$$
\int_{x Y^{-c}}^{x} e(\vartheta t) d\{t\} \ll\|\vartheta\| x \leq Y^{c} \ll \Psi(x, y) Y^{-c} \leq R(x, y ; \vartheta),
$$

ce qui est suffisant.

Nous avons

$$
\frac{y}{\log y}\left|\int_{\max \left\{x Y^{-c}, y\right\}}^{x} e(\vartheta t) \frac{\{t / y\}}{t} d t\right| \ll \frac{y \log (3 x / y)}{\log y} .
$$

D'autre part, une simple manipulation fournit

$$
\begin{aligned}
\int_{\max \left\{x Y^{-c}, y\right\}}^{x} e(\vartheta t) \frac{\{t / y\}}{t} d t & =\int_{\max \left\{x Y^{-c}, y\right\}}^{x} e(\vartheta t) \frac{d t}{y} \\
& -\sum_{\max \left\{x Y^{-c} / y, 1\right\} \leq k \leq x / y} \int_{k y}^{\min \{x,(k+1) y\}} k \frac{e(\vartheta t)}{t} d t \\
& \ll \frac{1}{\|\vartheta\| y} \log (x\|\vartheta\|+2) .
\end{aligned}
$$

Il en découle que

$$
\frac{y}{\log y}\left|\int_{x Y^{-c}}^{x} e(\vartheta t) \frac{\{t / y\}}{t} d t\right| \ll R(x, y ; \vartheta) .
$$

Posant

$$
\lambda_{y}(u):=\int_{0}^{u-1}\left(\varrho^{\prime}(u-v)+\frac{\varrho^{\prime \prime}(u-v)}{\log y}\right) \frac{\left\{y^{v}\right\}}{y^{v}} d v,
$$

nous évaluons $\int_{x Y_{-c}}^{x} e(\vartheta t) \lambda_{y}(u(t)) d t$ grâce à une intégration par parties. On a $\lambda_{y}(v)=0$ lorsque $v \leq 1$. Il vient

$$
\begin{aligned}
\int_{x Y^{-c}}^{x} e(\vartheta t) \lambda_{y}(u(t)) d t= & {\left[\frac{e(\vartheta t)-1}{2 \pi i\|\vartheta\|} \lambda_{y}(u(t))\right]_{\max \left\{x Y^{-c}, y\right\}}^{x} } \\
& -\int_{\max \left\{x Y^{-c}, y\right\}}^{x} \frac{\lambda_{y}^{\prime}(u(t))}{t \log y} \cdot \frac{e(\vartheta t)-1}{2 \pi i\|\vartheta\|} d t .
\end{aligned}
$$


La majoration

$$
\varrho^{(k)}(u-v) \ll \varrho(u)(\log (u+1))^{k} e^{v \xi(u)} \quad(k \in \mathbb{N})
$$

avec $\xi(u)=\log (u+1)+O(1)$ implique

$$
\int_{0}^{u-1} \varrho^{(k)}(u-v) \frac{\left\{y^{v}\right\}}{y^{v}} d v \ll \varrho(u)(\log (u+1))^{k} \int_{0}^{u-1} e^{v(\xi(u)-\log y)} d v .
$$

Pour $(x, y)$ satisfaisant à $\left(G_{\varepsilon}\right)$, pour $y$ suffisamment grand, on a par exemple $\xi(u) \leq(\log y) / 2$. Nous en déduisons que

$$
\int_{0}^{u-1} \varrho^{(k)}(u-v) \frac{\left\{y^{v}\right\}}{y^{v}} d v \ll \frac{\varrho(u)(\log (u+1))^{k}}{\log y} .
$$

Nous avons donc la majoration $\lambda_{y}(u) \ll \varrho(u) \log (u+1) / \log y$ et, par conséquent, grâce à (23),

$$
\left[\frac{e(\vartheta t)-1}{2 \pi i\|\vartheta\|} \lambda_{y}(u(t))\right]_{\max \left\{x Y^{-c}, y\right\}}^{x} \ll R(x, y ; \vartheta) .
$$

La dérivée de $\lambda_{y}$ s'écrit pour $u \geq 1$

$$
\lambda_{y}^{\prime}(u)=\int_{0}^{u-1}\left(\varrho^{\prime \prime}(u-v)+\frac{\varrho^{\prime \prime \prime}(u-v)}{\log y}\right) \frac{\left\{y^{v}\right\}}{y^{v}} d v-\frac{\left\{y^{u-1}\right\}}{y^{u-1}}\left(1-\frac{1}{\log y}\right) .
$$

En utilisant (23) et (30), nous obtenons à partir de (29)

$$
\begin{aligned}
& \int_{\max \left\{x Y^{-c}, y\right\}}^{x} e(\vartheta t) \lambda_{y}(u(t)) d t \\
& \ll x \varrho(u) \frac{(\log (u+1))^{2}}{(\log y)^{2}} \min \left\{1, \frac{\log (\|\vartheta\| x+2)}{\|\vartheta\| x}\right\}+\frac{\Psi(x, y)}{Y^{c}}+R_{1}(x, y, \vartheta)
\end{aligned}
$$

avec

$$
R_{1}(x, y ; \vartheta)=\int_{\max \left\{x Y^{-c}, y\right\}}^{x} \min \left\{t,\|\vartheta\|^{-1}\right\} \frac{y\{t / y\}}{t^{2} \log y} d t .
$$

Il ne reste donc plus qu'à majorer $R_{1}(x, y ; \vartheta)$. D'une part, on a

$$
R_{1}(x, y ; \vartheta) \leq \int_{y}^{x} \frac{y\{t / y\}}{t \log y} d t \ll \frac{y}{\log y} \log (3 x / y) .
$$

D'autre part, on a

$$
R_{1}(x, y ; \vartheta) \leq \frac{1}{\|\vartheta\|} \int_{y}^{x} \frac{y}{t^{2} \log y} d t \ll \frac{1}{\|\vartheta\| \log y} .
$$

En distinguant le cas $u \leq 2$ et le cas $u \geq 2$, nous obtenons donc

$$
R_{1}(x, y ; \vartheta) \ll R(x, y ; \vartheta) .
$$


En rassemblant ces résultats, nous avons bien l'estimation annoncée à la Proposition 2.

2.2. Démonstration du Théorème 2. De manière classique (voir [7] et [14]), nous nous ramenons à des sommes d'exponentielles. Soit

$$
A(\vartheta):=\sum_{a \in \mathcal{A}} e(a \vartheta), \quad B(\vartheta):=\sum_{b \in \mathcal{B}} e(b \vartheta) .
$$

Nous utilisons de manière fondamentale la formule

$$
\sum_{\substack{n \in \mathcal{A} \oplus \mathcal{B} \\ P(n) \leq y}} 1=\int_{0}^{1} A(\vartheta) B(\vartheta) E(2 x, y ;-\vartheta) d \vartheta
$$

issue de la relation $\int_{0}^{1} e(n \vartheta) d \vartheta=0$ si $n \neq 0$. Grâce au Théorème 1 , on a donc

$$
\begin{aligned}
\sum_{\substack{n \in \mathcal{A} \oplus \mathcal{B} \\
P(n) \leq y}} 1= & \varrho\left(\frac{\log (2 x)}{\log y}\right) \int_{0}^{1} A(\vartheta) B(\vartheta) E(2 x, 2 x ;-\vartheta) d \vartheta \\
& +O\left(\int_{0}^{1}|A(\vartheta)| \cdot|B(\vartheta)| \cdot|\Delta(2 x, y ;-\vartheta)| d \vartheta\right) \\
= & \varrho\left(\frac{\log (2 x)}{\log y}\right)|\mathcal{A}| \cdot|\mathcal{B}|+O\left(x \frac{\varrho(u) \log (u+1)}{\log y} \int_{0}^{1}|A(\vartheta)| \cdot|B(\vartheta)| d \vartheta\right) .
\end{aligned}
$$

Par l'inégalité de Cauchy-Schwarz, il vient

$$
\int_{0}^{1}|A(\vartheta)| \cdot|B(\vartheta)| d \vartheta \leq \sqrt{\int_{0}^{1}|A(\vartheta)|^{2} d \vartheta \int_{0}^{1}|B(\vartheta)|^{2} d \vartheta}=\sqrt{|\mathcal{A}| \cdot|\mathcal{B}|} .
$$

La relation

$$
\varrho\left(\frac{\log (2 x)}{\log y}\right)=\varrho(u)\left\{1+O\left(\frac{\log (u+1)}{\log y}\right)\right\}
$$

valable pour $(x, y)$ satisfaisant à $\left(G_{\varepsilon}\right)$ permet alors de conclure la démonstration.

2.3. Une autre application du Théorème 1. Pour clore ce travail, nous énonçons une autre conséquence du Théorème 1 . Soit

$$
\begin{aligned}
& S\left(N ; y_{1}, y_{2}, y_{3}\right) \\
& \quad:=\left|\left\{\left(n_{1}, n_{2}, n_{3}\right): n_{i} \geq 1, P\left(n_{i}\right) \leq y_{i}, N=n_{1}+n_{2}+n_{3}\right\}\right| .
\end{aligned}
$$

Dans [3], A. Balog et A. Sárközy montrent que pour tout entier $N \geq N_{0}$ on a $S\left(N ; y_{1}, y_{2}, y_{3}\right) \geq 1$ dès que

$$
y:=\min y_{i} \geq \exp \left\{3 \sqrt{\log N \log _{2} N}\right\} .
$$


Lorsque $y \geq \exp \left\{(1+\varepsilon) \log N \log _{3} N / \log _{2} N\right\}$, nous établissons une formule asymptotique de $S\left(N ; y_{1}, y_{2}, y_{3}\right)$.

Proposition 2. Soient $N \geq 3$ et des entiers $y_{1}, y_{2}, y_{3}$ tels que $y_{i} \in$ $[2, N]$. Posant $u_{i}:=\log N / \log y_{i}$, on a

$$
S\left(N ; y_{1}, y_{2}, y_{3}\right)=(1+o(1)) \frac{N^{2}}{2} \varrho\left(u_{1}\right) \varrho\left(u_{2}\right) \varrho\left(u_{3}\right) \quad(N \rightarrow \infty)
$$

uniformément par rapport à $y_{i}$ lorsque

$$
y:=\min y_{i} \geq \exp \left\{(1+\varepsilon) \frac{\log N \log _{3} N}{\log _{2} N}\right\} .
$$

Il est décevant de ne pas pouvoir étendre cette estimation pour $(N, y)$ satisfaisant à $\left(G_{\varepsilon}\right)$. Il est à noter qu'étant donné le domaine considéré, pour démontrer cette estimation, nous n'avons besoin que des estimations de $E(x, y ; \vartheta)$ établies dans [8]. Nous ne détaillons pas la démonstration qui est semblable à celle du Théorème 2 .

\section{Bibliographie}

[1] A. Balog and A. Sárközy, On sums of sequences of integers, I, Acta Arith. 44 (1984), 73-86.

[2] - - - On sums of sequences of integers, II, Acta Math. Hungar. 44 (1984), 169-179.

[3] - - - On sums of integers having small prime factors, I, Studia Sci. Math. Hungar. 19 (1984), 35-47.

[4] R. de la Bretèche, Sommes d'exponentielles et entiers sans grand facteur premier, Proc. London Math. Soc. (3) 77 (1998), 39-78.

[5] Y. Dupain, R. R. Hall et G. Tenenbaum, Sur l'équirépartition modulo 1 de certaines fonctions de diviseurs, J. London Math. Soc. (2) 26 (1982), 397-411.

[6] P. D. T. A. Elliott and A. Sárközy, The distribution of the number of prime divisors of sums $a+b$, J. Number Theory 29 (1988), 94-99.

[7] P. Erdős, H. Maier and A. Sárközy, On the distribution of the number of prime factors of sums $a+b$, Trans. Amer. Math. Soc. 302 (1987), 269-280.

[8] E. Fouvry et G. Tenen baum, Entiers sans grand facteur premier en progressions arithmétiques, Proc. London Math. Soc. (3) 63 (1991), 449-494.

[9] A. Hildebrand, On the number of the positive integers $\leq x$ and free of prime factors $>y$, J. Number Theory 22 (1986), 289-307.

[10] A. Hildebrand and G. Tenenbaum, Integers without large prime factors, J. Théor. Nombres Bordeaux 5 (1993), 411-484.

[11] E. Saias, Sur le nombre d'entiers sans grand facteur premier, J. Number Theory 32 (1989), 78-99.

[12] A. Sárközy and C. L. Stewart, On divisors of sums of integers, II, J. Reine Angew. Math. 365 (1986), 171-191.

[13] - - - On the average value of the numbers of divisors of sums $a+b$, Illinois J. Math. 38 (1984), 1-18. 
[14] G. Tenenbaum, Facteur premier de sommes d'entiers, Proc. Amer. Math. Soc. 106 (1989), 287-296.

[15] - Introduction à la théorie analytique et probabiliste des nombres, 2ème éd., Cours Spécialisé, no. 1, Soc. Math. France, 1995.

[16] I. M. Vinogradov, The Method of Trigonometrical Sums in Theory of Numbers, Interscience, New York, 1954.

Département de Mathématiques

Université de Paris XI-Orsay

Bâtiment 425

91405 Orsay Cedex, France

E-mail: Regis.La-Breteche@math.u-psud.fr

Reçu le 6.6.1997

et révisé le 20.3.1998 